\title{
MicroRNA-145 inhibits the malignant phenotypes of gastric carcinoma cells via downregulation of fascin 1 expression
}

\author{
MINGHUI XUE ${ }^{1}$, LUNDE ZHAO $^{1}$, FANG YANG $^{2}$, ZHENJUAN $^{2} I^{2}$ and GUANGYAN LI ${ }^{2}$ \\ Departments of ${ }^{1}$ General Surgery and ${ }^{2}$ Gastroenterology, The First Affiliated Hospital of Xinxiang Medical University, \\ Weihui, Henan 453100, P.R. China
}

Received January 21, 2015; Accepted November 5, 2015

DOI: $10.3892 / \mathrm{mmr} .2015 .4609$

\begin{abstract}
MicroRNA (miR)-145 has been demonstrated to act as a tumor suppressor, and deregulation of fascin 1 (FSCN1) has been observed in several types of human malignancy, including gastric carcinoma. However, the molecular mechanism underlying the function of miR-145, specifically its targets in gastric carcinoma have yet to be fully elucidated. In the present study, downregulation of miR-145 and upregulation of FSCN1 was identified in gastric carcinoma cell lines, compared with normal gastric mucosal epithelial cells. A luciferase reporter assay demonstrated that miR-145 was able to bind to the 3'-untranslated region of FSCN1 mRNA. Overexpression of miR-145 led to a significant decrease in FSCN1 expression levels, whereas knockdown of miR-145 resulted in increased FSCN1 expression levels in gastric carcinoma cells. Furthermore, overexpression of miR-145 inhibited proliferation, migration and invasion in gastric carcinoma cells. Similar effects were also observed in gastric carcinoma cells transfected with FSCN1 small interfering RNA. In addition, overexpression of FSCN1 reversed the suppressive effects of miR-145 upregulation on proliferation, migration and invasion in gastric carcinoma cells, suggesting that FSCN1 is indeed involved in the miR-145-mediated malignant phenotype of gastric carcinoma cells. The present study revealed an anti-oncogenic role of miR-145 in gastric carcinoma via inhibition of FSCN1, and suggested that miR-145 may be used for the treatment of gastric carcinoma.
\end{abstract}

\section{Introduction}

Gastric cancer is one of the most common types of human cancer worldwide (1). Although significant progress has been achieved in recent years, the early diagnosis and treatment

Correspondence to: Dr Minghui Xue, Department of General Surgery, The First Affiliated Hospital of Xinxiang Medical University, 88 Jiankang Road, Weihui, Henan 453100, P.R. China E-mail: xinxiangxueminghui@163.com

Key words: gastric carcinoma, microRNA-145, fascin 1, proliferation, migration, invasion for gastric cancer is not yet satisfactory. Furthermore, gastric carcinoma is difficult to cure due to its heterogeneity, therefore the prognosis remains poor (1). Investigation into the molecular mechanisms underlying gastric carcinoma have begun to yield results (2).

MicroRNAs (miRs) are a type of small non-coding RNA, that are able to regulate various physiological and developmental processes by mediating the expression levels of their target genes, via direct binding to the 3'-untranslated region (3'-UTR) of their target mRNAs (3). Furthermore, miRs have been demonstrated to be associated with tumorigenesis and tumor progression. miRs can promote or inhibit the development and progression of human cancer $(4,5)$. Deregulation of certain miRNAs, such as miR-10b, miR-29a, miR-145, miR-126, miR-133, miR-143, miR-148a, miR-218, miR-941, miR-1247 and miR-145, have been reported to be associated with gastric carcinoma (6-15). miR-145 has been shown to generally act as a tumor suppressor in numerous types of human cancers, such as colorectal cancer, gastric carcinoma, bladder cancer and glioma (14,16-20). Takagi et al (14) demonstrated that miR-145 was downregulated in the majority of the 43 gastric cancer tissue samples examined. Qiu et al (13) suggested that miR-145 suppressed the proliferation, migration, invasion and cell cycle progression of gastric cancer cells by targeting transcription factor Sp1. As one miRNA may target various mRNAs, other targets may also be involved in miR-145-mediated malignant phenotypes of gastric carcinoma cells.

The present study aimed to reveal the regulatory mechanism by which miR-145 mediates the malignant phenotype of gastric cancer cells, focusing on its target genes.

\section{Materials and methods}

Materials. Dulbecco's modified Eagle's medium (DMEM), TRIzol reagent, fetal bovine serum (FBS), an miRNA Reverse Transcription kit, a SYBR Ex Taq kit, and Lipofectamine 2000 were purchased from Invitrogen (Thermo Fisher Scientific, Inc., Waltham, MA, USA). An miRNA Q-Polymerase Chain Reaction (PCR) Detection kit was purchased from GeneCopoeia (Rockville, MD, USA). Mouse anti-human FSCN1 monoclonal antibody (dilution, 1:500; cat. no. ab49815), mouse anti-human GAPDH monoclonal antibody (dilution, 1:500; cat. no ab184531) and rabbit anti-mouse 
IgG secondary antibody (dilution, 1:10,000; cat. no. ab6728) were purchased from Abcam (Cambridge, UK). An Enhanced Chemiluminescence (ECL) kit was purchased from Pierce Biotechnology, Inc. (Rockford, IL, USA). A Quick-Change Site-Directed Mutagenesis kit was purchased from Agilent Technologies, Inc. (Santa Clara, CA, USA). A PsiCHECK 2 vector was purchased from Promega Corporation (Madison, WI, USA), and a Migration Detection kit I was purchased from BD Biosciences (San Jose, CA, USA).

Cell culture. Five human gastric cancer cell lines, BGC823, SGC7901, SNU5, HGC27 and AGS cells, as well as the GES1 normal gastric mucosa epithelial cell line were purchased from the Cell Bank of the Type Culture Collection of the Chinese Academy of Sciences (Shanghai, China). The cells were cultured in DMEM supplemented with $10 \%$ FBS, $100 \mathrm{IU} / \mathrm{ml}$ penicillin (Beyotime Institute of Biotechnology, Wuhan, China) and $100 \mathrm{mg} / \mathrm{ml}$ streptomycin (Beyotime Institute of Biotechnology) incubated at $37^{\circ} \mathrm{C}$ in a humidified chamber containing $5 \% \mathrm{CO}_{2}$.

Reverse transcription-quantitative (RT-q)PCR assay. Total RNA was extracted using TRIzol reagent, according to the manufacturer's instructions. For the detection of the mRNA expression of FSCN1, a RevertAid First-Strand cDNA Synthesis kit (Thermo Fisher Scientific, Inc., Pittsburgh, PA, USA) was used to reverse transcribe RNA into cDNA, according to the manufacturer's instructions. mRNA expression was detected using the SYBR Green qPCR Assay kit, according to the manufacturer's protocol. GAPDH served as an endogenous reference. The specific primers were as follows: FSCN1 forward, 5'-CCAGGGTATGGA CCTGTCTG-3', and reverse, 5'-GTGTGGGTACGGAAG GCAC-3'; GAPDH forward, 5-GGAGCGAGATCCCTC CAAAAT-3', and reverse, 5'-GGCTGTTGTCATACTTCT CATGG-3'. The reaction conditions were as follows: $95^{\circ} \mathrm{C}$ for $3 \mathrm{~min}$, followed by 40 cycles of denaturation at $95^{\circ} \mathrm{C}$ for $15 \mathrm{sec}$ and annealing/elongation at $60^{\circ} \mathrm{C}$ for $30 \mathrm{sec}$. The PCR reaction was performed on a 7500 Real-Time PCR System (Applied Biosystems; Thermo Fisher Scientific, Inc.). For the detection of miR-145 expression, a miRNA Reverse Transcription kit was used to reverse transcribe RNA into cDNA, according to the manufacturer's instructions. The expression levels of miR-145 were then evaluated using a miRNA Q-PCR Detection kit, following the manufacturer's protocol. The U6 small nuclear RNA was used for normalization. The relative mRNA and miRNA expression levels were analyzed by the $2^{-\Delta \Delta C q}$ method.

Western blotting. Western blotting was used to examine relative protein expression levels. Briefly, total protein was extracted using radioimmunoprecipitation assay buffer (Beyotime Institute of Biotechnology), and separated by $12 \%$ sodium dodecyl sulfate-polyacrylamide gel electrophoresis (Beyotime Institute of Biotechnology), and then transferred onto a polyvinylidene difluoride (PVDF) membrane (EMD Millipore, Billerica, MA, USA). The PVDF membrane was then incubated with Tris-buffered saline with Tween 20 (Beyotime Institute of Biotechnology) containing 5\% milk at room temperature for $3 \mathrm{~h}$ for blocking. The membrane was subsequently incubated with mouse anti-FSCN1, and mouse anti-GAPDH primary antibodies at room temperature for $3 \mathrm{~h}$. Following washing with phosphate-buffered saline (PBS) with Tween 20 three times, the PVDF membrane was incubated with rabbit anti-mouse secondary antibodies at room temperature for $1 \mathrm{~h}$. Chemiluminescence detection was performed using the ECL kit. The relative protein expression levels were analyzed using Image-Pro plus software 6.0 (Media Cybernetics, Inc., Rockville, MD, USA), and the values were presented as the density ratio compared with GAPDH.

Transfection. For functional analysis, transfection was performed using Lipofectamine 2000 according to the manufacturer's instructions. For FSCN1 functional analysis, cells were transfected with FSCN1-specific small interfering (si)RNA (produced by Auragene Biosciences, Changsha, China) or pcDNA3.1-FSCN1 plasmids (produced by Auragene Biosciences). For miR-145 functional analysis, cells were transfected with scrambled miRNA (negative control), miR-145 mimics or miR-145 inhibitor (all produced by Auragene Biosciences)

Luciferase reporter assay. Following to the manufacturer's instructions, a mutant type 3'-UTR of FSCN1 was generated using the Quick-Change Site-Directed Mutagenesis kit. The wild or mutant type 3'-UTR of FSCN1 was then inserted into the psiCHECK2 vector using the restriction endonucleases, XhoI and $\mathrm{NotI}$ at the multiple cloning regions in the psiCHECK2 vector. A luciferase reporter assay was subsequently performed. Briefly, the cells were cultured $\left(37^{\circ} \mathrm{C}\right.$ in a humidified chamber containing $5 \% \mathrm{CO}_{2}$ ) to $70 \%$ confluence with or without $100 \mathrm{nM}$ miR-145 mimics, prior to being transfected with psiCHECK 2-FSCN1-3'-UTR or psiCHECK 2-mutant FSCN1-3'-UTR vector. Following incubation at $37^{\circ} \mathrm{C}$ in an atmosphere containing $5 \% \mathrm{CO}_{2}$ for $48 \mathrm{~h}$, luciferase activity levels were determined using an LD400 luminometer (Beckman Coulter, Inc., Brea, CA, USA). Renilla luciferase activity was normalized to firefly luciferase activity.

MTT assay. An MTT assay (Beyotime Institute of Biotechnology) was performed to examine cell proliferation. Briefly, for each group (cells transfected with miR-145 mimics, FSCN1 siRNA or co-transfected with miR-145 mimics and FSCN1 plasmid), $1 \times 10^{4}$ cells/well were plated in a 96-well plate, and incubated for $48 \mathrm{~h}$ at $37^{\circ} \mathrm{C}$ in an atmosphere containing $5 \% \mathrm{CO}_{2}$. To assess cell proliferation, $10 \mu \mathrm{l} \mathrm{MTT}$ $(5 \mathrm{mg} / \mathrm{ml}$ ) was added to each well, and further incubated for $4 \mathrm{~h}$ at $37^{\circ} \mathrm{C}$ in an atmosphere containing $5 \% \mathrm{CO}_{2}$. The supernatant was removed, and $100 \mu$ l dimethyl sulfoxide was added to dissolve the precipitation. The absorbance was detected at $492 \mathrm{~nm}$ using a microplate reader (Bio-Rad Laboratories, Inc., Hercules, CA, USA).

Scratch assay. A scratch assay was performed to determine the cell migratory capacity in each group. Cells were cultured to full confluence $(70 \%)$ at $37^{\circ} \mathrm{C}$ in a humidified chamber containing $5 \% \mathrm{CO}_{2}$, and a scratch wound of $\sim 1 \mathrm{~mm}$ width was created with a plastic scriber. The cells were then washed with PBS, and cultured at $37^{\circ} \mathrm{C}$ in an atmosphere containing 
A

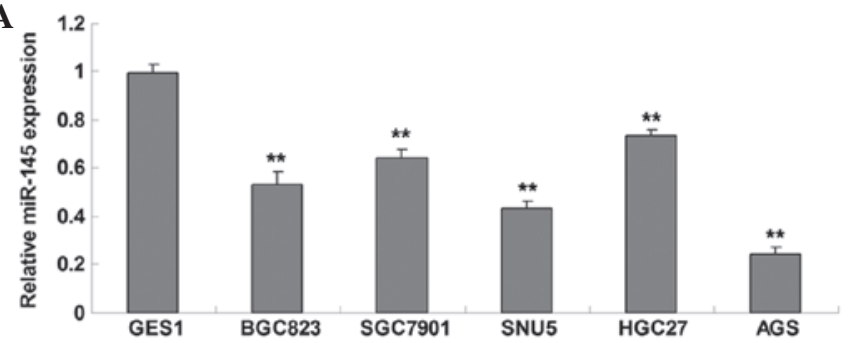

B

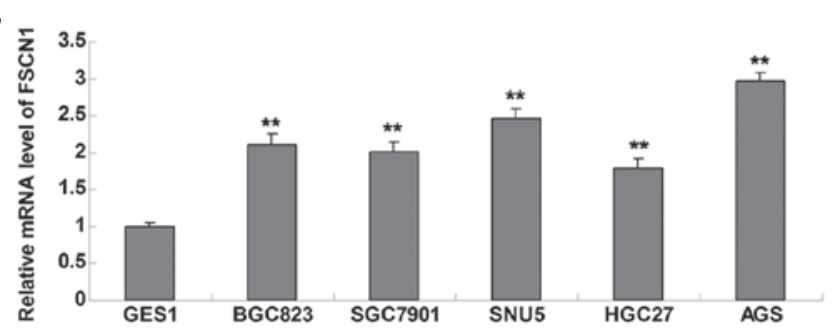

Figure 1. (A) Reverse transcription-quantitative polymerase chain reaction was performed to examine the relative expression levels of miR-145 in five gastric carcinoma cell lines, as well as in a GES1 normal gastric mucosa epithelial cell line, which served as a control. ${ }^{* *} \mathrm{P}<0.01$, vs. the GES1 cells. (B) Quantification of western blot analysis was performed to examine the relative protein expression levels of FSCN1 in five gastric carcinoma cell lines, as well as in a GES1 normal gastric mucosa epithelial cell line, which served as a control. GAPDH was used as an internal reference. ${ }^{* *} \mathrm{P}<0.01$, vs. the GES1 cells. GAPDH, glyceraldehyde 3-phosphate dehydrogenase; miR, microRNA; FSCN1, fascin 1.

A

B

$\begin{array}{rr}\text { FSCN13'-UTR } & \text { AGAAAAUGACCAAA } \\ \text { miR-133a } & \text { ACUUCCCCUGGUUI II } \\ \text { mutant FSCN13'-UTR } & \text { AGAAAAUGAGGUAA }\end{array}$

C

D
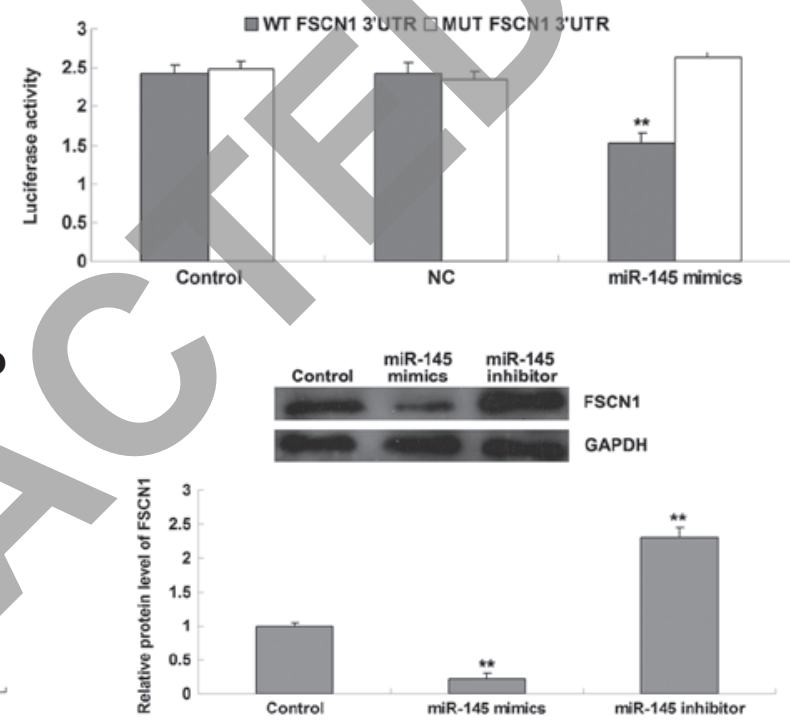

Figure 2. (A) Seed sequences of miR-145 in the WT and MUT 3'-UTR of FSCN1. (B) A luciferase reporter assay demonstrated that the luciferase activity levels were significantly reduced in AGS cells co-transfected with miR-145 mimics and WT 3'-UTR of FSCN1, but remained unchanged in AGS cells co-transfected with miR-145 mimics and MUT FSCN1 3'-UTR, as compared with the control group. AGS cells co-transfected with blank vector and WT FSCN1 3'-UTR or MUT FSCN1 3'-UTR served as controls. (C) Reverse transcription-quantitative polymerase chain reaction was performed to examine the relative expression levels of miR-145 in AGS cells transfected with miR-145 mimics or inhibitor. AGS cells that did not receive any treatment served as control cells. (D) Western blot analysis was performed to examine the relative protein expression levels of FSCN1 in AGS cells transfected with miR-145 mimics or inhibitor. GAPDH was used as an internal reference. AGS cells without any transfection served as control cells. ${ }^{* *} \mathrm{P}<0.01$, vs. the control. GAPDH, glyceraldehyde 3 -phosphate dehydrogenase; WT, wild-type; NC, negative control; MUT, mutant-type; FSCN1, fascin 1; 3'-UTR, 3'-untranslated region; miR, microRNA.

$5 \% \mathrm{CO}_{2}$ for $48 \mathrm{~h}$. The cells in each group were then fixed and observed under a microscope (CKX41; Olympus Corporation, Tokyo, Japan.

Invasion assay. A Transwell assay was conducted for invasion analysis (using Matrigel-coated polyethylene terephthalate membrane chambers; BD Biosciences), and a cell suspension containing $5 \times 10^{5}$ cells $/ \mathrm{ml}$ was prepared in serum-free DMEM. A total of $300 \mu \mathrm{l}$ cell suspension was then added into the upper chamber and $500 \mu \mathrm{l}$ RPMI-1640 (Invitrogen: Thermo Fisher Scientific, Inc.) supplemented with $10 \%$ FBS was added into the lower chamber. Following incubation for $24 \mathrm{~h}$, non-invading cells as well as the matrix gel (BD Biosciences) on the interior of the inserts was removed using a cotton-tipped swab. Invasive cells on the lower surface of the membrane were stained using $0.1 \%$ crystal violet (Beyotime Institute of Biotechnology) for
$20 \mathrm{~min}$, and then rinsed with water and dried. Five fields were randomly selected, and cell number was counted under a microscope (CKX41; Olympus Corporation).

Statistical analysis. The values are presented as the mean \pm standard deviation of three independent experiments. Statistical analysis of differences was performed by one-way analysis of variance using SPSS 17.0 software (SPSS, Inc., Chicago, IL, USA). $\mathrm{P}<0.05$ was considered to indicate a statistically significant difference.

\section{Results}

Expression levels of miR-145 and FSCN1 in gastric carcinoma cells. RT-qPCR was conducted to determine the expression level of miR-145 in five common gastric cancer cell lines, 
A
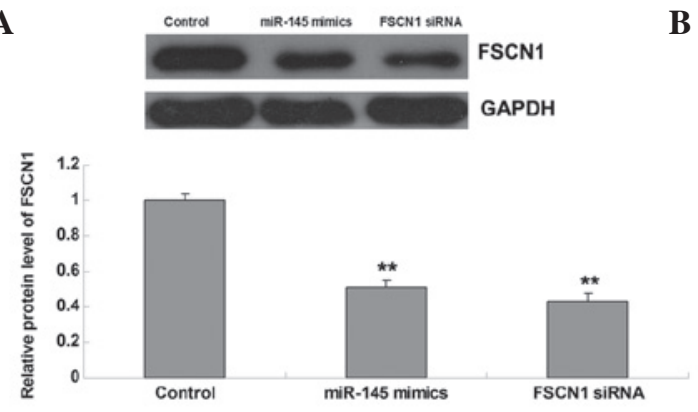

C
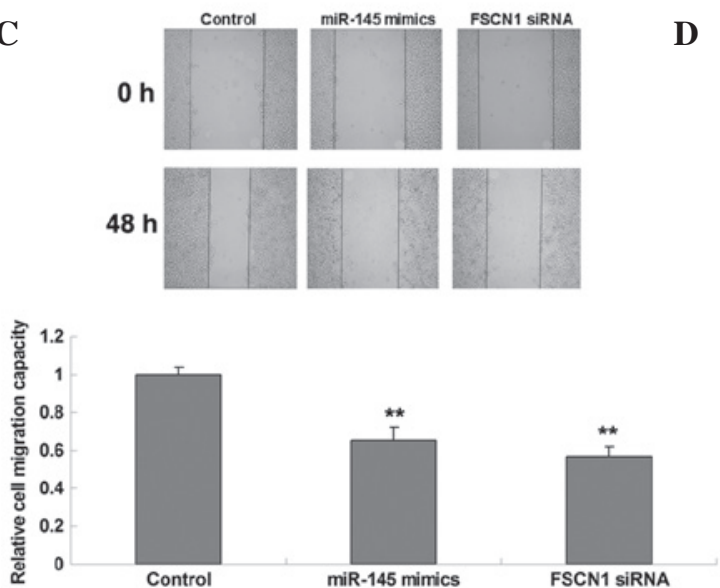

B

D
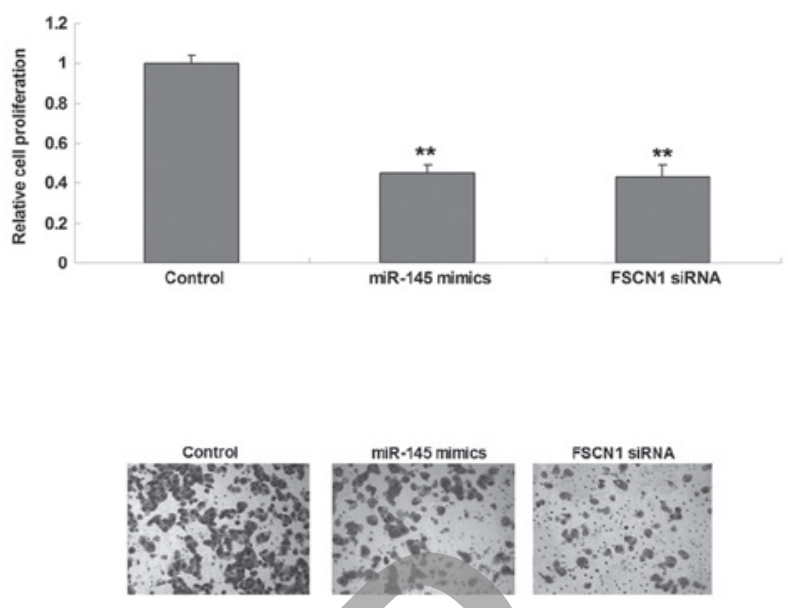

.

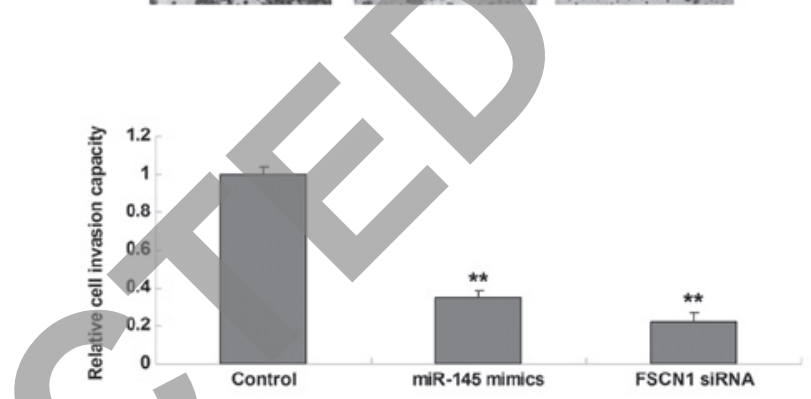

Figure 3. (A) Western blot analysis was performed to examine the relative protein expression levels of FSCN1 in AGS cells transfected with miR-145 mimics or FSCN1 siRNA. GAPDH was used as an internal reference. (B) An MTT assay was performed to examine the cell proliferation of AGS cells transfected with miR-145 mimics or FSCN1 siRNA. (C) Scratch assay was performed to examine the migratory capacity of AGS cells transfected with miR-145 mimics or FSCN1 siRNA. (D) A Transwell assay was performed to examine the invasive capacity of AGS cells transfected with miR-145 mimics or FSCN1 siRNA. AGS cells without any transfection served as a control. ${ }^{* *} \mathrm{P}<0.01$, vs. the control. GAPDH, glyceraldehyde 3-phosphate dehydrogenase; FSCN1, fascin 1; siRNA, small interfering RNA; miR, microRNA.

BGC823, SGC7901, SNU5, HGC27 and AGS, as well as in the GES1 normal gastric mucosa epithelial cell line, which served as a control. As shown in Fig. 1A, miR-145 was significantly downregulated in gastric cancer cell lines, as compared with GES1 normal gastric mucosa epithelial cells. The expression levels of FSCN1 were subsequently examined in the five common gastric cancer cell lines and normal gastric mucosa epithelial cells by RT-qPCR. The protein expression levels of FSCN1 were significantly upregulated in gastric carcinoma cell lines, as compared with those in GES1 normal gastric mucosa epithelial cells (Fig. 1B). These results suggest that miR-145 is downregulated whereas FSCN1 is upregulated in gastric carcinoma cells. In addition, as AGS cells exhibited the greatest changes in miR-145 and FSCN1 expression levels, this cell line was used in the following experiments of this study.

miR-145 suppresses FSCN1 expression by binding to the 3'-UTR of FSCN1 mRNA in gastric carcinoma cells. The putative seed sequences for miR-145 at the 3'-UTR of FSCN1 are shown in Fig. 2A. To clarify whether or not miR-145 was able to bind to the 3'-UTR of FSCN1 mRNA, a wild type and mutant type FSCN1 3'-UTR were generated (Fig. 2A), and a luciferase reporter assay was subsequently performed in the AGS gastric carcinoma cells. Luciferase activity levels were significantly reduced in AGS cells with the wild type 3'-UTR of FSCN1 mRNA co-transfected with miR-145 mimics; however, luciferase activity levels did not change in AGS cells with mutant type 3'-UTR of FSCN1 mRNA co-transfected with miR-145 mimics, compared with the control group (Fig. 2B), indicating that FSCN1 is a direct target gene of miR-145. It was further investigated whether miR-145 could affect the expression levels of FSCN1 in gastric carcinoma cells. Following transfection with miR-145 mimics and inhibitor into AGS cells, RT-qPCR was performed to determine the changes in miR-145 expression levels. As shown in Fig. 2C, transfection with miR-145 mimics led to significant upregulation of miR-145 expression levels, whereas transfection with an miR-145 inhibitor resulted in reduced miR-145 expression levels in AGS cells. In addition, the protein expression levels of FSCN1 were reduced in AGS cells overexpressing miR-145, and these expression levels were increased following knockdown of miR-145 (Fig. 2D). Therefore, the results suggest that miR-145 negatively regulates the protein expression of FSCN1 in AGS gastric carcinoma cells, perhaps by directly binding to the 3'-UTR of FSCN1 mRNA.

Effects of miR-145 and FSCN1 on proliferation, migration and invasion in gastric carcinoma cells. The roles of miR-145 and FSCN1 in the regulation of malignant phenotypes of gastric cancer cells were further investigated. AGS cells were transfected with miR-145 mimics or FSCN1 siRNA. Post-transfection, the expression levels of FSCN1 in each group were quantified, and showed that transfection 
A
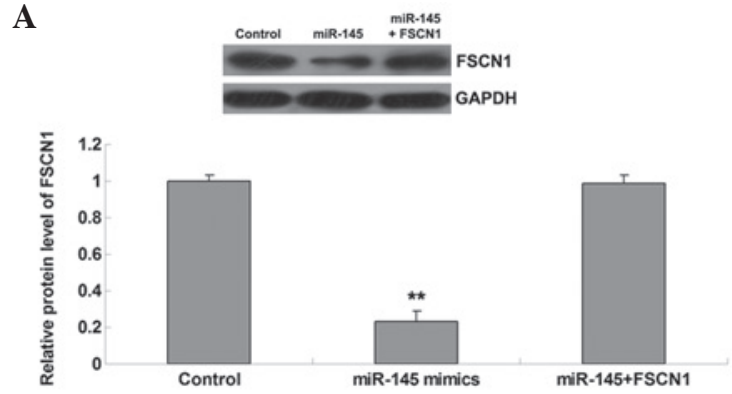

C ${ }^{\text {Control }}$
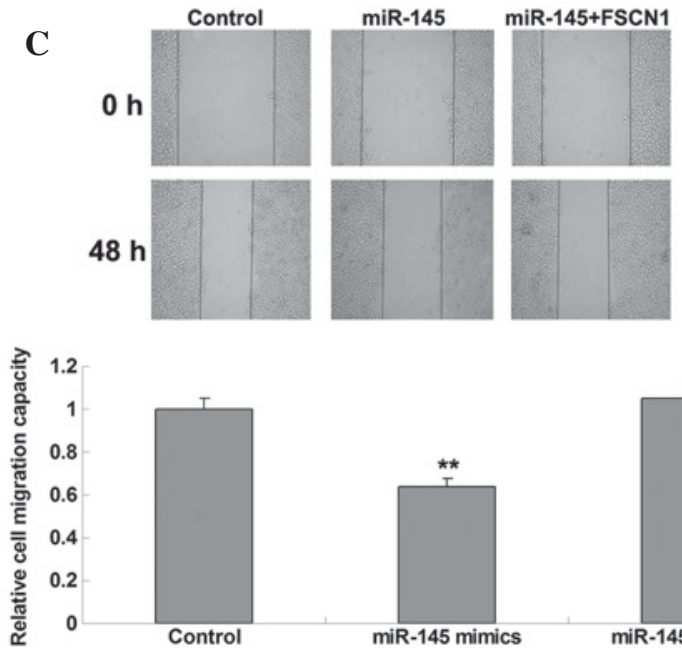

B

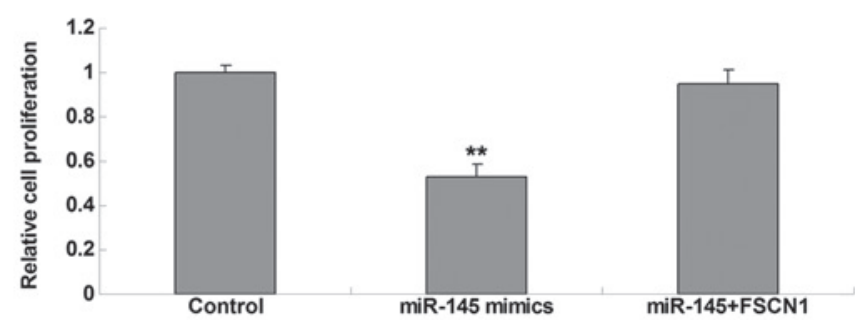

D

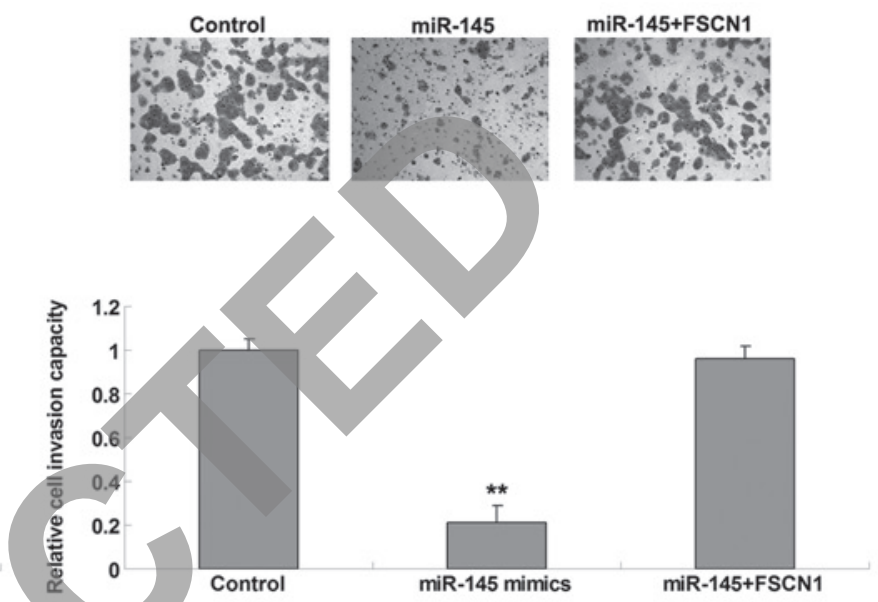

Figure 4. (A) Western blot analysis was performed to examine the relative protein expression levels of FSCN1 in AGS cells transfected with miR-145 mimics or co-transfected with miR-145 mimics and FSCN1 plasmids. (B) An MTT assay was performed to examine the proliferation of AGS cells transfected with miR-145 mimics or co-transfected with miR-145 mimics and FSCN1 plasmid. AGS cells without any transfection served as a control. ${ }^{* * *} \mathrm{P}<0.01$, vs. the control. (C) Scratch assay was performed to examine the migratory capacity of AGS cells transfected with miR-145 mimics or co-transfected with miR-145 mimics and FSCN1 plasmids. (D) A Transwell assay was performed to determine the invasion capacity of AGS cells transfected with miR-145 mimics or co-transfected with miR-145 mimics and FSCN1 plasmids. AGS cells without any transfection served as a control. ${ }^{* *} \mathrm{P}<0.01$, vs. the control. GAPDH, glyceraldehyde 3-phosphate dehydrogenase; FSCN1, fascin 1; siRNA, small interfering RNA; miR, microRNA.

with miR-145 mimics and FSCN1 siRNA reduced FSCN1 expression levels (Fig. 3A). Subsequently, cell proliferation, migration and invasion analyses were performed. As shown in Fig. 3B-D, overexpression of miR-145 inhibited proliferation, migration and invasion, similar to the effects of FSCN1 knockdown.

FSCN1 overexpression reverses the inhibitory effects of miR-145 upregulation on proliferation, migration and invasion in gastric carcinoma cells. To further clarify whether FSCN1 was involved in miR-145-mediated malignant phenotypes of gastric carcinoma cells, AGS cells were transfected with miR-145 mimics, or co-transfected with miR-145 mimics and pcDNA3.1-FSCN1 plasmids. The protein expression levels of FSCN1 were subsequently determined in each group. As shown in Fig. 4A, co-transfection with miR-145 mimics and FSCN1 plasmids reversed the inhibitory effect on FSCN1 expression in AGS cells compared with transfection with miR-145 mimics alone. The proliferation, migration and invasion of these two groups were also compared. As shown in Fig. 4B-D, overexpression of FSCN1 reversed the suppressive effects of miR-145 upregulation on proliferation, migration and invasion in AGS cells, indicating that FSCN1 is indeed involved in miR-145-mediated malignant phenotypes of gastric cancer cells.

\section{Discussion}

MicroRNAs inhibit the protein expression of their target genes by binding to the 3'-UTR of target mRNAs based on sequence complementarity (3). The present study identified FSCN1 as a target of miR-145 in gastric carcinoma cells, and demonstrated that miR-145 was downregulated, whereas FSCN1 was upregulated in gastric carcinoma cell lines, as compared with normal gastric mucosal epithelial cells. Furthermore, the results suggested that the suppressive effects of miR-145 on proliferation, migration and invasion in gastric carcinoma cells occur partly through direct inhibition of FSCN1 expression.

Deregulation of miRs has been demonstrated to be associated with tumorigenesis and tumor progression (21). In the present study, miR-145 was demonstrated to be significantly downregulated in gastric carcinoma cell lines, as compared with normal gastric mucosal epithelial cells, results which were concordant with those of previous studies that demonstrated that the expression levels of miR-145 were reduced in gastric carcinoma tissue samples and cell lines $(13,14)$. Downregulated expression levels of miR-145 have also been reported in other types of cancer including glioma, colorectal cancer, and esophageal squamous cell carcinoma (22-25). It has been reported that miR-145 targets the transcription factor SP1, the knockdown of which inhibits the expression of matrix 
metalloproteinase-9 and cyclin D1 associated with cell growth and invasion in gastric carcinoma cells (13). Furthermore, overexpression of miR-145 induced higher sensitivity of gastric cancer cells to 5-fluorouracil, and the possible candidate targets of miR-145 were identified to be insulin receptor substrate-1 and $\beta$-actin (14). The results of the present study demonstrated that overexpression of miR-145 inhibited cell proliferation, migration and invasion in gastric carcinoma cells. However, the molecular mechanism by which miR-145 mediates the malignant phenotypes of gastric carcinoma cells remains to be elucidated.

Furthermore, the results of the present study demonstrated that FSCN1 was a target gene of miR-145 in gastric cancer cells, and was negatively regulated by miR-145. FSCN1, a member of the FSCN family of actin-binding proteins, has an important role in the organization of F-actin into parallel bundles and in the formation of filopodia $(26,27)$. In addition, FSCN1 participates in the regulation of cellular interactions, adhesion and motility, and overexpression of FSCN1 is associated with cancer metastasis by promoting cell motility $(27,28)$. Recently, deregulation of FSCN1 was shown to occur in gastric carcinoma (29). Tsai et al (30) demonstrated that $>50 \%$ of the 60 poorly differentiated gastric adenocarcinoma tissue samples exhibited moderate or strong FSCN1 expression, and higher expression levels of FSCN1 were directly correlated with more-advanced cancer stages, and inversely correlated with survival rate, suggesting that aberrant upregulation of FSCN1 may be involved in the progression of gastric adenocarcinoma (30). In the present study, the results demonstrated that the expression levels of FSCN1 were upregulated in gastric carcinoma cells, compared with normal gastric mucosal epithelial cells, and knockdown of FSCN1 inhibited AGS cell proliferation, migration and invasion. A previous study also showed that inhibition of FSCN1 expression suppressed the proliferation and metastasis of gastric carcinoma cells (31). In addition, FSCN1 was involved in the transforming growth factor- $\beta 1$-induced invasion and metastasis in gastric carcinoma (32).

The results of the present study demonstrated that FSCN1 was involved in miR-145-mediated AGS cell proliferation, migration and invasion. The association between miR-145 and FSCN1 has also been demonstrated in several other types of cancer $(23,33)$. For instance, miR-145 was found to suppress tumor cell invasion and migration by targeting FSCN1 in breast cancer cells (33). In addition, miR-145 inhibited proliferation and invasion via the suppression of FSCN1 in esophageal squamous cell carcinoma cells (24). The expression of FSCN1 is also mediated by other miRs in several types of cancer. For example, Akanuma et al (34) demonstrated that knockdown of FSCN1 inhibited the proliferation and invasion of esophageal squamous cell carcinoma cells, similar to the effect of miR-133a overexpression, and identified FSCN1 as a target of miR-133a. Another study suggested that upregulated miR-451 in colon cancer cells may inhibit AMP-activated protein kinase from activating mammalian target of rapamycin complex 1, which mediates FSCN1 expression and cancer cell progression (35).

In conclusion, the results of the present study revealed an anti-oncogenic role of miR-145 in gastric cancer via direct inhibition of its target gene FSCN1. Therefore, the present study suggested that miR-145 may be used for the treatment of gastric carcinoma.

\section{References}

1. Ishiguro H, Kimura M and Takeyama H: Role of microRNAs in gastric cancer. World J Gastroenterol 20: 5694-5699, 2014.

2. Kuo CY, Chao Y and Li CP: Update on treatment of gastric cancer. J Chin Med Assoc 77: 345-353, 2014

3. Bartel DP: MicroRNAs: Genomics, biogenesis, mechanism, and function. Cell 116: 281-297, 2004.

4. Ambros V: The functions of animal microRNAs. Nature 431: 350-355, 2004.

5. Baer C, Claus R and Plass C: Genome-wide epigenetic regulation of miRNAs in cancer. Cancer Res 73: 473-477, 2013

6. Xia J, Guo X, Yan J and Deng K: The role of miR-148a in gastric cancer. J Cancer Res Clin Oncol 140: 1451-1460, 2014.

7. Chen L, Xiao H, Wang ZH, Huang Y, Liu ZP, Ren H and Song H: miR-29a suppresses growth and invasion of gastric cancer cells in vitro by targeting VEGF-A. BMB Rep 47: 39-44, 2014.

8. Feng R, Chen X, Yu Y, Su L, Yu B, Li J, Cai Q, Yan M, Liu B and Zhu Z: miR-126 functions as a tumour suppressor in human gastric cancer. Cancer Lett 298: 50-63, 2010.

9. He XP, Shao Y, Li XL, Xu W, Chen GS, Sun HH, Xu HC, Xu X, Tang D, Zheng XF, et al: Downregulation of miR-145 in gastric cancer correlates with cyclooxygenase- 2 overexpression and tumor growth. FEBS J 279: 4201-4212, 2012.

10. Gao P, Xing AY, Zhou GY, Zhang TG, Zhang JP, Gao C, Li H and Shi DB: The molecular mechanism of microRNA-145 to suppress invasion-metastasis cascade in gastric cancer. Oncogene 32: 491-501, 2013.

11. Kim JG, Kim TO, Bae JH, Shim JW, Kang MJ, Yang K, Ting AH and Yi JM: Epigenetically regulated MIR941 and MIR1247 target gastric cancer cell growth and migration. Epigenetics 9: 1018-1030, 2014.

12. Kim K, Lee HC, Park JL, Kim M, Kim SY, Noh SM, Song KS, Kim JC and Kim YS: Epigenetic regulation of microRNA-10b and targeting of oncogenic MAPRE1 in gastric cancer. Epigenetics 6: 740-751, 2011.

3. Qiu T, Zhou X, Wang J, Du Y, Xu J, Huang Z, Zhu W, Shu Y and Liu P: MiR-145, miR-133a and miR-133b inhibit proliferation, migration, invasion and cell cycle progression via targeting transcription factor Sp1 in gastric cancer. FEBS Lett 588: 1168-1177, 2014.

14. Takagi T, Iio A, Nakagawa Y, Naoe T, Tanigawa N and Akao Y: Decreased expression of microRNA-143 and -145 in human gastric cancers. Oncology 77: 12-21, 2009.

15. Tie J, Pan Y, Zhao L, Wu K, Liu J, Sun S, Guo X, Wang B, Gang Y,Zhang Y, et al: MiR-218 inhibits invasion and metastasis of gastric cancer by targeting the Robol receptor. PLoS Genet 6: e1000879, 2010

16. Wang CJ, Zhou ZG, Wang L, Yang L, Zhou B, Gu J, Chen HY and Sun XF: Clinicopathological significance of microRNA-31, -143 and -145 expression in colorectal cancer. Dis Markers 26: 27-34, 2009.

17. Ni Y, Meng L, Wang L, Dong W, Shen H, Wang G, Liu Q and Du J: MicroRNA-143 functions as a tumor suppressor in human esophageal squamous cell carcinoma. Gene 517: 197-204, 2013.

18. Wang Y, Hu C, Cheng J, Chen B, Ke Q, Lv Z, Wu J and Zhou Y: MicroRNA-145 suppresses hepatocellular carcinoma by targeting IRS1 and its downstream Akt signaling. Biochem Biophys Res Commun 446: 1255-1260, 2014.

19. Rani SB, Rathod SS, Karthik S, Kaur N, Muzumdar D and Shiras AS: MiR-145 functions as a tumor-suppressive RNA by targeting Sox 9 and adducin 3 in human glioma cells. Neuro Oncol 15: 1302-1316, 2013.

20. Zhang H, Pu J, Qi T, Qi M, Yang C, Li S, Huang K, Zheng L and Tong Q: MicroRNA-145 inhibits the growth, invasion, metastasis and angiogenesis of neuroblastoma cells through targeting hypoxia-inducible factor 2 alpha. Oncogene 33: 387-397, 2014.

21. Croce CM and Calin GA: miRNAs, cancer, and stem cell division. Cell 122: 6-7, 2005.

22. Lee HK, Bier A, Cazacu S, Finniss S, Xiang C, Twito H, Poisson LM, Mikkelsen T, Slavin S, Jacoby E, et al: MicroRNA-145 is downregulated in glial tumors and regulates glioma cell migration by targeting connective tissue growth factor. PLoS One 8: e54652, 2013.

23. Feng Y, Zhu J, Ou C, Deng Z, Chen M, Huang W and Li L: MicroRNA-145 inhibits tumour growth and metastasis in colorectal cancer by targeting fascin-1. Br J Cancer 110: 2300-2309, 2014. 
24. Kano M, Seki N, Kikkawa N, Fujimura L, Hoshino I, Akutsu Y, Chiyomaru T, Enokida $\mathrm{H}$, Nakagawa $\mathrm{M}$ and Matsubara $\mathrm{H}$ : miR-145, miR-133a and miR-133b: Tumor-suppressive miRNAs target FSCN1 in esophageal squamous cell carcinoma. Int J Cancer 127: 2804-2814, 2010.

25. Speranza MC, Frattini V, Pisati F, Kapetis D, Porrati P, Eoli M, Pellegatta S and Finocchiaro G: NEDD9, a novel target of miR-145, increases the invasiveness of glioblastoma. Oncotarget 3: 723-734, 2012.

26. Yang S, Huang FK, Huang J, Chen S, Jakoncic J, Leo-Macias A, Diaz-Avalos R, Chen L, Zhang JJ and Huang XY: Molecular mechanism of fascin function in filopodial formation. J Biol Chem 288: 274-284, 2013.

27. Machesky LM and Li A: Fascin: Invasive filopodia promoting metastasis. Commun Integr Biol 3: 263-270, 2010.

28. Yamakita Y, Matsumura F, Lipscomb MW, Chou PC, Werlen G, Burkhardt JK and Yamashiro S: Fascin1 promotes cell migration of mature dendritic cells. J Immunol 186: 2850-2859, 2011.

29. Guo L, Bai H, Zou D, Zou D, Hong T, Liu J, Huang J, He P, Zhou Q and He J: The role of microRNA-133b and its target gene FSCN1 in gastric cancer. J Exp Clin Cancer Res 33: 99, 2014.

30. Tsai WC, Jin JS, Chang WK, Chan DC, Yeh MK, Cherng SC, Lin LF, Sheu LF and Chao YC: Association of cortactin and fascin-1 expression in gastric adenocarcinoma: Correlation with clinicopathological parameters. J Histochem Cytochem 55: 955-962, 2007
31. Fu H, Wen JF, Hu ZL, Luo GQ and Ren HZ: Knockdown of fascin 1 expression suppresses the proliferation and metastasis of gastric cancer cells. Pathology 41: 655-660, 2009.

32. Fu H, Hu Z, Wen J, Wang K and Liu Y: TGF-beta promotes invasion and metastasis of gastric cancer cells by increasing fascin1 expression via ERK and JNK signal pathways. Acta Biochim Biophys Sin (Shanghai) 41: 648-656, 2009.

33. Chiyomaru T, Enokida H, Tatarano S, Kawahara K, Uchida Y, Nishiyama K, Fujimura L, Kikkawa N, Seki N and Nakagawa M: miR-145 and miR-133a function as tumour suppressors and directly regulate FSCN1 expression in bladder cancer. Br J Cancer 102: 883-891, 2010.

34. Akanuma N, Hoshino I, Akutsu Y, Murakami K, Isozaki Y, Maruyama T, Yusup G, Qin W, Toyozumi T, Takahashi M, et al: MicroRNA-133a regulates the mRNAs of two invadopodia-related proteins, FSCN1 and MMP14, in esophageal cancer. Br J Cancer 110: 189-198, 2014.

35. Chen MB, Wei MX, Han JY, Wu XY, Li C, Wang J, Shen W and Lu PH: MicroRNA-451 regulates AMPK/mTORC1 signaling and fascin1 expression in HT-29 colorectal cancer. Cell Signal 26: 102-109, 2014. 